\title{
Visible light optical coherence tomography measures retinal oxygen metabolic response to systemic oxygenation
}

\author{
Ji Yi $^{1}$, Wenzhong Liu ${ }^{1}$, Siyu Chen ${ }^{1}$, Vadim Backman ${ }^{1}$, Nader Sheibani ${ }^{2}$, Christine M. Sorenson ${ }^{2}$, Amani A. Fawzi ${ }^{3}$, \\ Robert A. Linsenmeier ${ }^{1,3,4}$ and Hao F. Zhang ${ }^{1,3}$
}

The lack of capability to quantify oxygen metabolism noninvasively impedes both fundamental investigation and clinical diagnosis of a wide spectrum of diseases including all the major blinding diseases such as age-related macular degeneration, diabetic retinopathy, and glaucoma. Using visible light optical coherence tomography (vis-OCT), we demonstrated accurate and robust measurement of retinal oxygen metabolic rate $\left(\mathrm{rMRO}_{2}\right)$ noninvasively in rat eyes. We continuously monitored the regulatory response of oxygen consumption to a progressive hypoxic challenge. We found that both oxygen delivery, and $\mathrm{rMRO}_{2}$ increased from the highly regulated retinal circulation $(\mathrm{RC})$ under hypoxia, by $0.28 \pm 0.08 \mu \mathrm{L} \mathrm{\textrm {min } ^ { - 1 }}(p<0.001)$, and $0.20 \pm 0.04 \mu \mathrm{L} \mathrm{min}^{-1}(p<0.001)$ per $100 \mathrm{mmHg}$ systemic $\mathrm{pO}_{2}$ reduction, respectively. The increased oxygen extraction compensated for the deficient oxygen supply from the poorly regulated choroidal circulation. Results from an oxygen diffusion model based on previous oxygen electrode measurements corroborated our in vivo observations. We believe that vis-OCT has the potential to reveal the fundamental role of oxygen metabolism in various retinal diseases.

Light: Science \& Applications (2015) 4, e334; doi:10.1038/lsa.2015.107; published online 25 September 2015

Keywords: oxygen metabolism; retinal circulation; visible light optical coherence tomography

\section{INTRODUCTION}

Retinal cell degeneration is ubiquitous in the early stages of essentially all the major blinding retinal diseases (e.g., loss of pericytes in diabetic retinopathy $(D R)^{1}$, retinal ganglion cells in glaucoma ${ }^{2}$, retinal pigment epithelial (RPE) cells in age-related macular degeneration $)^{3}$. Because the highly metabolic retinal cells rely on sufficient oxygen supply to maintain their functions, abnormality of retinal oxygen metabolism naturally could cause cell degeneration ${ }^{4}$. This aberrant oxygen metabolism can further create prolonged impact on retinal function through oxygen-sensitive signaling pathways ${ }^{5-7}$, such as hypoxia-induced factor-mediated pathways ${ }^{8}$. Therefore, when accurately measured, the retinal oxygen metabolic rate $\left(\mathrm{rMRO}_{2}\right)$ could potentially be a sensitive biomarker for early-stage diagnosis or an indicator for progression of several retinal diseases.

Currently there is no single non-invasive technique to measure $\mathrm{rMRO}_{2}$ in vivo. Direct measurement of intraretinal oxygen tension $\left(\mathrm{PO}_{2}\right)$ using microelectrodes is considered as the gold standard in quantifying oxygen metabolism ${ }^{9}$; however, the procedure is invasive and difficult to practice. Two-photon phosphorescence imaging has also been applied to quantify intraretinal $\mathrm{PO}_{2}$, but it relies on contrast agents ${ }^{10}$. Magnetic resonance imaging can measure temporal changes of $\mathrm{PO}_{2}$ but only qualitatively in humans ${ }^{11}$. Alternatively, $\mathrm{rMRO}_{2}$ can be measured by quantifying the consumption of oxygen derived from the retinal circulation ( $\mathrm{RC}$ ), which requires concurrent quantification of both blood flow and hemoglobin oxygen saturation $\left(\mathrm{sO}_{2}\right)$. Several techniques such as laser Doppler velocimetry ${ }^{12-14}$, multi-wavelength fundus photography and laser scanning ophthalmoscopy ${ }^{15-18}$, and photoacoustic ophthalmoscopy ${ }^{19-20}$ have been demonstrated to measure one of the two parameters, but not both together. Optical coherence tomography (OCT) $)^{21}$ is another major technique for threedimensional (3D) retinal imaging. Different OCT methods such as phase-sensitive optical Doppler coherence tomography ${ }^{22-23}$ and optical microangiography ${ }^{24}$ have been introduced to measure retinal blood flow, but $\mathrm{rMRO}_{2}$ is still inaccessible without other independent $\mathrm{sO}_{2}$ measurements.

Here we demonstrate that visible light optical coherence tomography (vis-OCT) can quantify $\mathrm{rMRO}_{2}$ in vivo through concurrent measurement of blood flow and $\mathrm{sO}_{2}$ from $\mathrm{RC}$. We have previously demonstrated that $\mathrm{sO}_{2}$ can be measured in vivo through spectral analysis of OCT signals acquired within the visible light spectral range ${ }^{25}$. The key advantage of visOCT is that the $3 \mathrm{D}$ imaging capability allows one to recover optical spectra specifically from blood vessels and eliminate the confounding signal from other retinal layers ${ }^{25-27}$. We first validated the blood flow and $\mathrm{sO}_{2}$ measurements both in vitro and in vivo. Then, as a proof of principle, we investigated the metabolic response to progressive hypoxia challenges. The experimental results were cross-validated by an oxygen

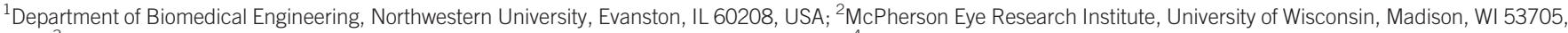

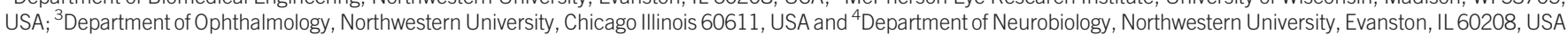
Correspondence: HF Zhang, Email: hfzhang@northwestern.edu

Received: 11 March 2015; revised: 1 June 2015; accepted: 7 June 2015; accepted article preview online 10 June 2015 
diffusion model derived from direct oxygen tension measurements in rat outer retina using microelectrodes.

\section{MATERIALS AND METHODS}

Visible light optical coherence tomography imaging system

Vis-OCT (Figure 1) uses a supercontinuum light source (SuperK, NKT photonics) with a working bandwidth from $520 \mathrm{~nm}$ to $630 \mathrm{~nm}$. The illuminating power on the cornea was measured to be $0.8 \mathrm{~mW}$, which is within the ANSI laser safety standard (Supplementary laser safety standard calculation). Briefly, we used a free-space interferometry configuration to minimize the dispersion, where the probing beam was collimated and split by a cube beam splitter into the reference and sample arms. The beam in the sample arm was steered by a pair of galvanometer mirrors to scan the focal point across the retina. The two beams from the reference and sample arms recombined at the beam splitter and were collected by an optical fiber. The fiber delivered the light to a home-made spectrometer that collected the interference spectral fringes by a line-scan CCD camera (spl2k, Basler). The full-width-half-maximum (FWHM) of the spectral coverage was $\sim 85 \mathrm{~nm}$, giving us $1.7-\mu \mathrm{m}$ axial resolution. The lateral resolution was estimated to be $15 \mu \mathrm{m}$ in the retina. Two scanning protocols were performed. Protocol 1 raster-scanned a $20^{\circ}$ squared retinal area with $256 \times 256$ pixels in each direction at a $25 \mathrm{kHz}$ A-line rate. Protocol 2 used dual circle scanning that scanned two concentric circles centered at the optic disk with 4096 pixels in each circle at a $70 \mathrm{kHz}$ A-line rate. The dual circle scan pattern was repeated eight times and the results were averaged to remove motion artifacts and pulsatile flow pattern. Protocol 1 was used for $\mathrm{sO}_{2}$ measurement and microvascular imaging. Protocol 2 was used for blood flow measurement, which requires much denser scanning. For visualizing the microvasculature, the mean intensity projection of a slab at depth range from $150 \mu \mathrm{m}$ to $200 \mu \mathrm{m}$ with respect to the retinal surface was taken to capture the microvasculature. The system schematic (Supplementary Fig. S1), scanning, data acquisition protocols, and data processing (Supplementary Fig. S2) are explained in detail in the Supplementary Information.

\section{Quantification of $\mathrm{rMRO}_{2}$ by vis-OCT}

To quantify $\mathrm{rMRO}_{2}$ (gas volume of oxygen consumed per unit time, $\mathrm{mL} \min ^{-1}$ ), we measured the overall oxygen difference between the major arterioles and venules in RC. The extracted oxygen from RC primarily supports inner retinal metabolism but could also supply part of the outer retina. Two parameters from the RC: total retinal blood flow $F\left(\mu \mathrm{L} \mathrm{min}{ }^{-1}\right)$ and $\mathrm{sO}_{2}(\%)$ were measured, and the $\mathrm{rMRO}_{2}$ was calculated according to:

$$
\mathrm{rMRO}_{2}=1.34 \times \mathrm{C}_{\mathrm{Hb}} \times F \times\left(s_{a} \mathrm{O}_{2}-s_{v} \mathrm{O}_{2}\right),
$$

where $C_{\mathrm{Hb}}$ is the total hemoglobin concentration $\left(\mathrm{g} \mu \mathrm{L}^{-1}\right) ; 1.34$ is the oxygen-binding capacity of hemoglobin $\left(\mathrm{mL} \mathrm{g}^{-1}\right)^{28} ; \mathrm{s}_{\mathrm{a}} \mathrm{O}_{2}$ and $\mathrm{s}_{\mathrm{v}} \mathrm{O}_{2}$ are arterial and venous $\mathrm{sO}_{2}$ with the subscript of $a$ and $v$ denoting arterial and venous blood, respectively. All major retinal arterioles branch (and venules merge) at the optic disk such that the global $\mathrm{rMRO}_{2}$ a

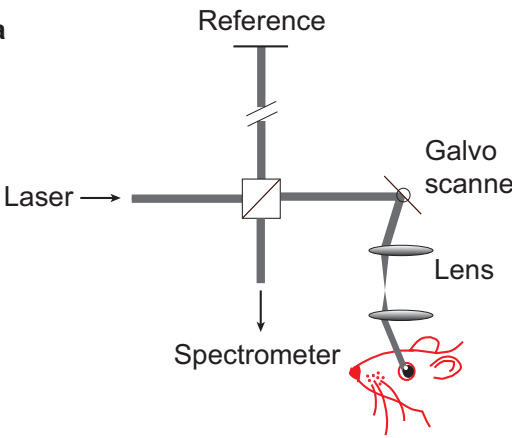

b

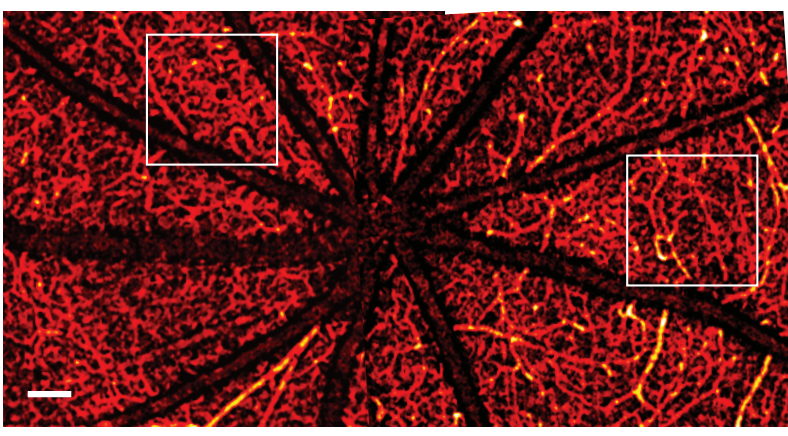

C

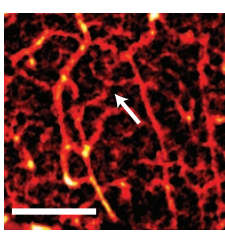

d

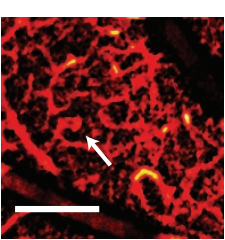

Figure 1 Principle of vis-OCT retinal imaging. (a) Illustration of vis-OCT system. A laser beam with a bandwidth from $520 \mathrm{~nm}$ to $630 \mathrm{~nm}$ was focused onto the retina. The reflected light interferes with the reference light, and the interference spectral fringes were collected by a home-built spectrometer. The interference spectral fringes were used to reconstruct the echo reflected from different depths of the retina. As the focal point was scanned across the retina by galvo mirrors, the 3D retinal structure was obtained. Further post-processing calculated the $\mathrm{rMRO}_{2}$ and produced the retinal microangiogram. (b) Example of a wide-field view of retinal microvasculature imaged by vis-OCT. (c-d) Magnified view of the highlight squared areas in the panel $\mathbf{b}$. The arrows highlight the smallest capillary vessels. Bar: $200 \mu \mathrm{m}$. 
a

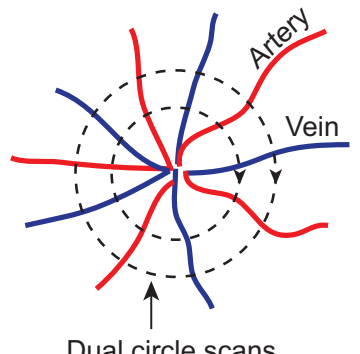

Dual circle scans b

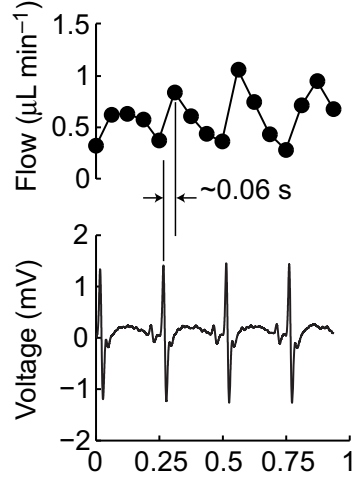

C

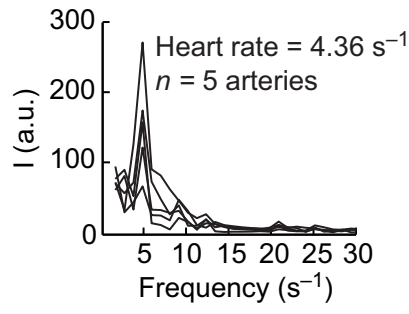

d

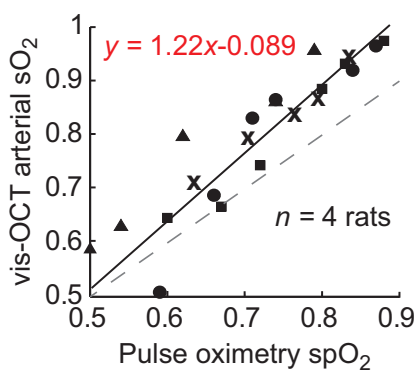

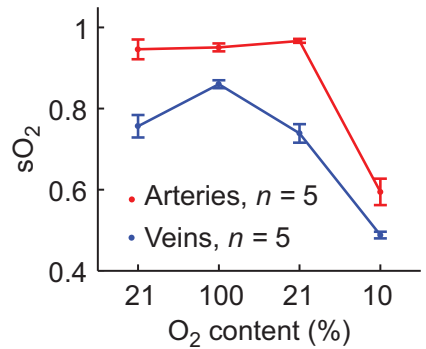

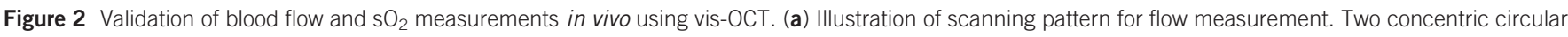

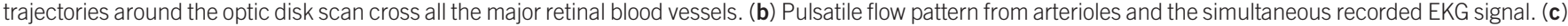

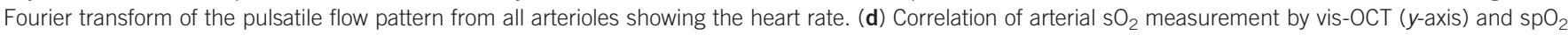

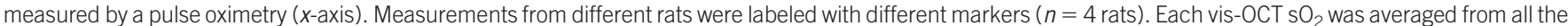

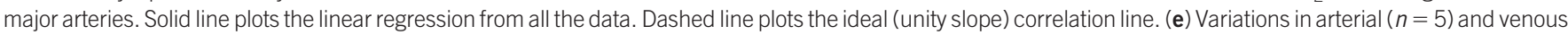
$(n=5) \mathrm{sO}_{2}$ responding to the changing oxygen content of the inhaled air. Error bar $=\mathrm{SEM}$.

derived from the $\mathrm{RC}$ can be calculated by measuring the flow and $\mathrm{sO}_{2}$ from major vessels around the optic disk.

\section{Blood flow measurement by phase sensitive Doppler OCT algorithm}

In phase sensitive Doppler OCT, interferometric spectral signals from two adjacent A-lines were used to extract blood velocity ${ }^{29}$. The blood velocity can be expressed as

$$
v=\frac{f_{\text {sample }} \cdot \lambda_{0} \cdot \Delta \phi}{4 \cdot \pi \cdot n \cdot \cos (\theta)}
$$

where $f_{\text {sample }}$ is the OCT A-line scanning frequency ${ }^{30} ; \lambda_{0}(\mathrm{~nm})$ is the center wavelength of the OCT light source; $\Delta \phi$ (radians) is the phase shift between adjacent A-scans after inverse Fourier transform; $n=$ 1.38 (dimensionless) is the refraction index of the sample; and $\theta$ (radians) is the Doppler angle: the angle between the blood vessel and probing light (Supplementary Fig. S3). Phase wrap was present when the blood velocity was high. With the knowledge of flow direction (inward or outward from the optic disk), we corrected the phase wrapping by adding or subtracting $2 \pi$ to or from the original phase shift. The Doppler angle $\theta$ was calculated by the relative displacement of each vessel between two circular scan images (Supplementary Information) $^{29,31}$.

Because of the higher axial resolution than lateral resolution in visOCT, we directly measured the vessel height $H$ axially from B-scan intensity images from the circular scanning protocol. The upper and lower boundaries of the vessels were manually selected.
After correcting the Doppler angle, the diameter of the blood vessel, Dia, is

$$
\operatorname{Dia}=H \times \sin (\theta) .
$$

The total blood flow is equal to the product of the velocity and the vessel cross-sectional area

$$
\text { Flow }=v \times \frac{\pi}{4} \mathrm{Dia}^{2}
$$

\section{Oxygen saturation rate $\left(\mathrm{sO}_{2}\right)$ measurement by spectroscopic OCT analysis}

A Gaussian window with FWHM $k_{w}=0.32 \mu \mathrm{m}^{-1}$ (17-nm bandwidth at 585-nm center wavelength) swept the interferometric spectrum to select sub spectral bands. After the inverse Fourier transform on each sub-band, spatially resolved spectra can be obtained at each $3 \mathrm{D}$ voxel. The ability to selectively collect light reflected from a particular depth was the key to avoid the background reflectance that has hampered the accuracy of fundus photography-based methods ${ }^{15}$. The spectral signal from the vessel bottom along the vessel center lines was used to fit the following analytical model based on a modified Beer's law:

$$
\begin{aligned}
I^{2}(\lambda)= & I_{0}^{2}(\lambda) R_{0} r(\lambda) \exp \left[-2 d \mu_{\mathrm{HbO}_{2}}(\lambda) \times s \mathrm{O}_{2}-2 d \mu_{\mathrm{Hb}}(\lambda)\right. \\
& \left.\times\left(1-s \mathrm{O}_{2}\right)\right],
\end{aligned}
$$

where $I_{0}(\lambda)$ is the incident intensity on the retina. We ignored the optical attenuation induced by the cornea, lens, and vitreous chamber due to their high transmission within the visible spectral range ${ }^{32}$ and, 
thus, took the source spectrum as $I_{0} ; R_{0}$ is the reference arm reflectance; $d(\mathrm{~mm})$ is the vessel diameter; $r(\lambda)$ (dimensionless) is the reflectance at the vessel wall, whose scattering spectrum can be modeled as a power law under the first-order Born approximation $r(\lambda)=$ $A \lambda^{-\alpha}$, where $A$ (dimensionless) is a constant. The optical attenuation coefficient $\mu\left(\mathrm{mm}^{-1}\right)$ combines the absorption $\left(\mu_{\mathrm{a}}\right)$ and scattering coefficients $\left(\mu_{\mathrm{s}}\right)$ of the whole blood, which are both wavelength- and oxygenation-dependent. The subscripts $\mathrm{Hb}$ and $\mathrm{HbO}_{2}$ denote the contribution from deoxygenated and oxygenated blood, respectively. A linear regression to the logarithmic spectra returned the value of $\mathrm{sO}_{2}$.

\section{Animal preparation}

All the experimental procedures were approved by the Northwestern University IACUC and conformed to the Association for Research in Vision and Ophthalmology Statement on Animal Research. Two anesthesia protocols were used in this work. For data shown in Figure 2, we initially anesthetized adult Long-Evans rats with 4.5\% isoflurane for $10 \mathrm{mins}$, followed by $2.5 \%$ isoflurane during the imaging session. Isofluorane provided deeper anesthesia and better animal stability during the experiment, which is preferable for technology validation purposes. However, isoflurane is known to introduce vasodilation which would affect physiological studies ${ }^{33}$. Thus, for data shown in Figures 3 and 4 that involved vessel size measurements, we anesthetized rats with a mixture of ketamine and xylazine (ketamine: $11.45 \mathrm{mg} \mathrm{mL}^{-1}$; xylazine: $1.7 \mathrm{mg} \mathrm{mL}^{-1}$, in saline) injected intraperitoneally. The initial injection used a dose of $10 \mathrm{~mL} \mathrm{~kg}^{-1}$ body weight, followed by a $0.5-\mathrm{mL}$ intraperitoneal injection every half an hour after the initial injection. A pulse oximeter was attached to the left rear paw of the animal to monitor its arterial $\mathrm{sO}_{2}$ and heart rate (Supplementary Table). The body temperature was maintained between $36.5^{\circ} \mathrm{C}$ and $37.5^{\circ} \mathrm{C}$ using a heating pad (homeothermic blanket system, Stoelting Co.) underneath the animal. A home-made three-lead electrocardiogram (ECG) was used to record cardiac cycles. The animal was placed on a custom-made animal holder for imaging. We applied 0.5\%
Tetracaine Hydrochloride ophthalmic solution to the rats' eyes for local anesthesia and applied 1\% Tropicamide Hydrochloride ophthalmic solution to dilate the pupil. Commercial artificial tears were applied to the rats' eyes every other minute to prevent corneal dehydration. The inhaled gas was a mixture of oxygen and nitrogen. The ratio of the two gases was controlled by a gas proportioner ( 7300 Series, Matheson). The ventilated gas was changed to a hypoxic mixture according to the protocols as shown in the section on "Results and discussion". After the hypoxia challenge, the animal was allowed to recover and stabilize under normal air $\left(21 \% \mathrm{O}_{2}\right)$ for $5 \mathrm{~min}$ before being released.

\section{Analytical model of oxygen consumption in the outer retina} Oxygen consumption is defined as volume of oxygen consumed by tissue per unit time (gas volume consumed per unit time). The units of $\mathrm{rMRO}_{2}$ and oxygen consumption are the same, but the distinction is that we refer to oxygen consumption as what the inner and/or outer retina actually uses, whereas $\mathrm{rMRO}_{2}$ is what the RC supplies.

Because the outer retina is avascular, oxygen is supplied solely through diffusion. An analytical model can be applied based on Fick's second law according to the $\mathrm{PO}_{2}$ at both sides of the outer retina and the geometry of the outer retina. This model has been validated by microelectrode measurements on different species, including rats, cats, and macaques ${ }^{34-36}$.

Oxygen diffusion in the outer retina can be described by a onedimensional three-layer diffusion model based on Fick's second law ${ }^{34}$

$$
Q O_{2}=D k \frac{d^{2} P}{d x^{2}}
$$

where $\mathrm{QO}_{2}$ is the oxygen consumption normalized by tissue weight $\left(\mathrm{mL} \mathrm{O}_{2} \bullet \mathrm{min}^{-1} \bullet 100 \mathrm{~g}^{-1}\right) ; \mathrm{D}$ is the diffusivity of oxygen in tissue $\left(1.97 \times 10^{-5} \mathrm{~cm}^{2} \mathrm{~s}^{-1}\right) ; k$ is the solubility of oxygen $\left(2.4 \times 10^{-5} \mathrm{~mL} \mathrm{O}_{2} /\right.$ $(\mathrm{mL}$ retina $\bullet \mathrm{mmHg})) ; \mathrm{P}$ is $\mathrm{PO}_{2}(\mathrm{mmHg})$; and $x$ is the distance from the choroid. The photoreceptor outer segments, which do not consume oxygen, occupy layer 1 in the model, between $x=0$ and $x=L_{1}$. The oxygen-consuming inner segments are between $x=L_{1}$ and $x=L_{2}$.

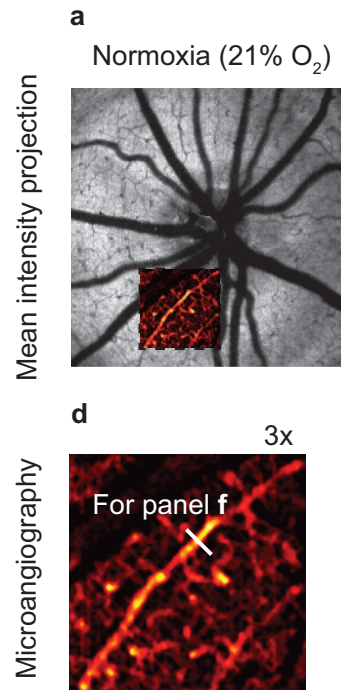

b

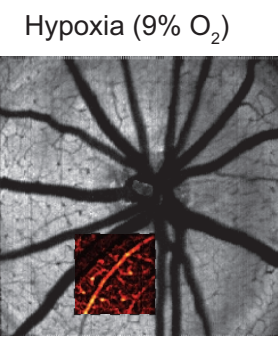

e

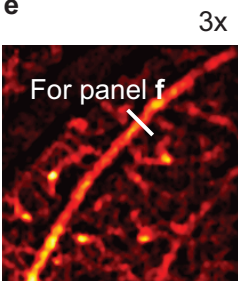

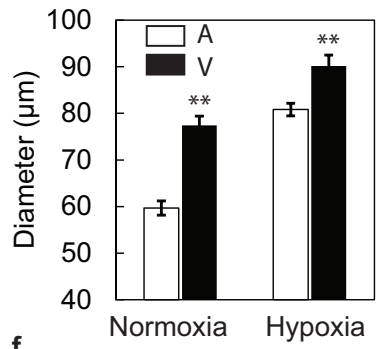

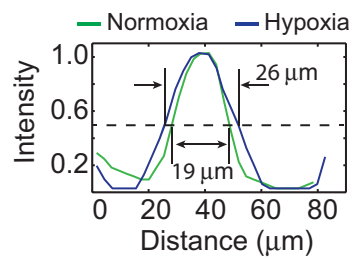

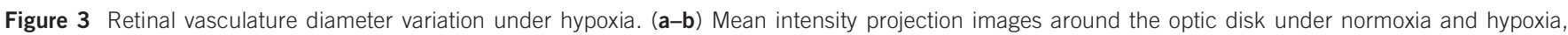

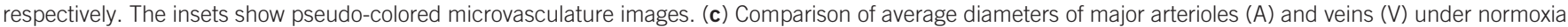

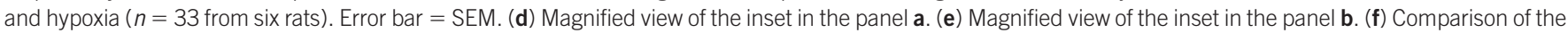
arteriole diameters highlighted in both panels $\mathbf{d}$ and $\mathbf{e}$ under normoxia and hypoxia. Bar: $200 \mu \mathrm{m}$. **p<0.01 from two sample $t$-test. 
a

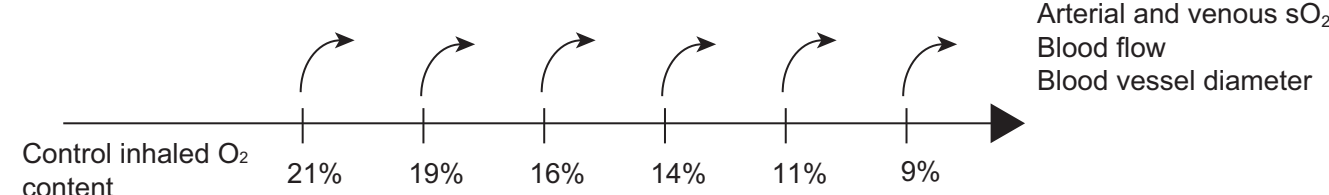

b

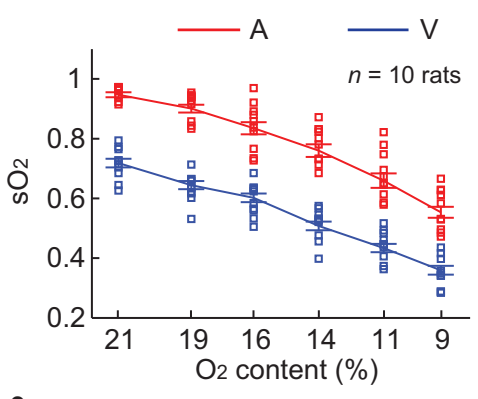

e

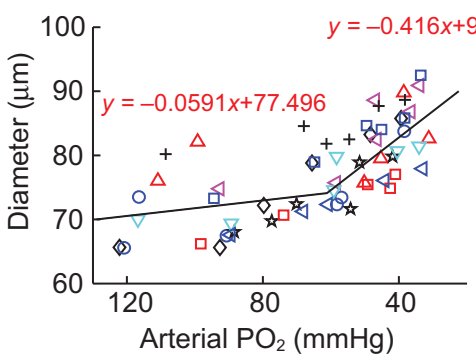

g

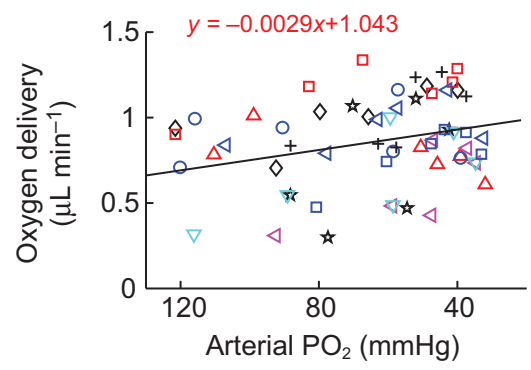

C

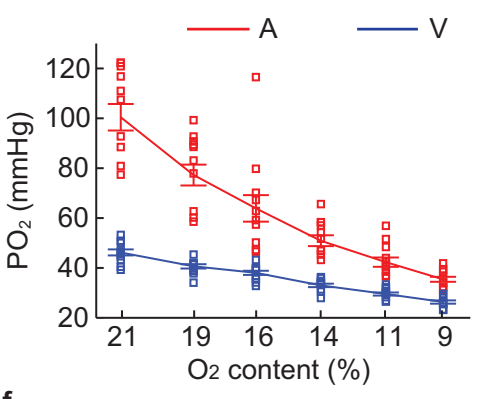

f

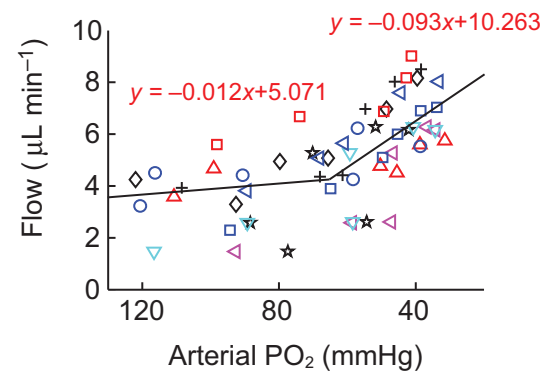

h

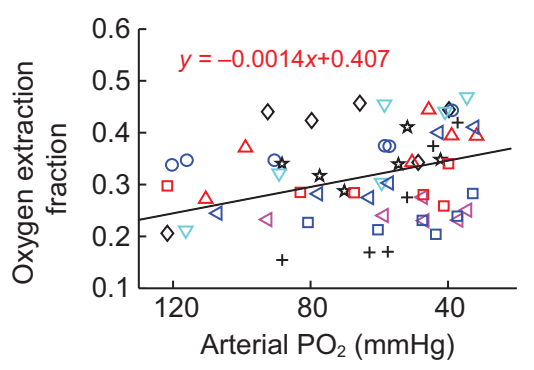

d

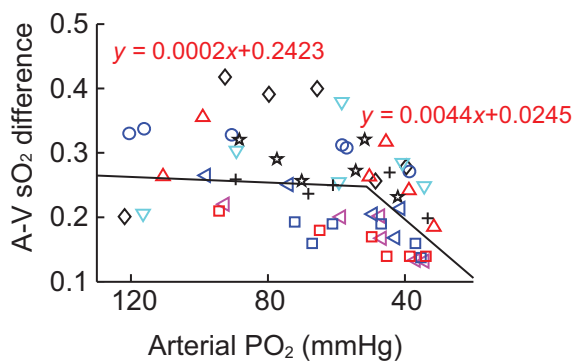

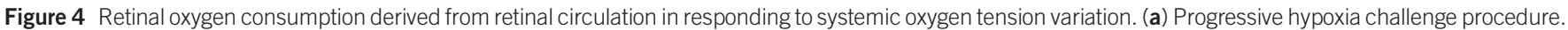

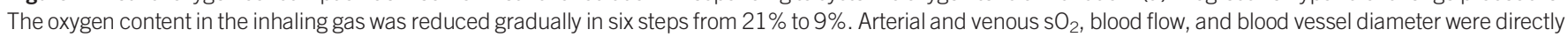

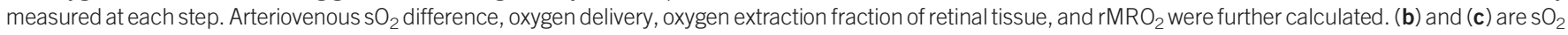

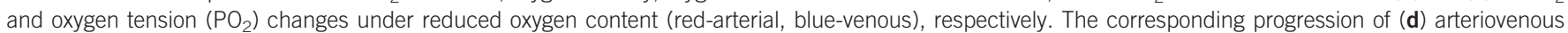

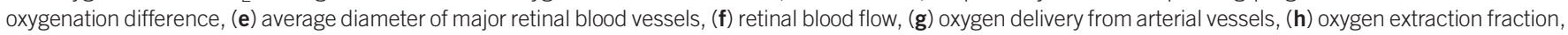

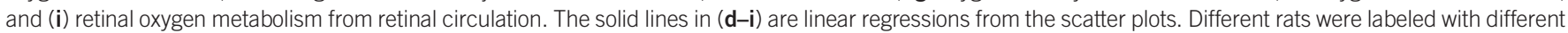
markers ( $n=10$ rats). In $\mathbf{i}$, regression lines for each rat are shown in the inset on the same axis as the main figure.

The third layer is the outer nuclear layer (photoreceptor cell bodies), which do not consume oxygen, and is between $x=L_{2}$ and $x=L$, where $L$ is the thickness of the outer retina. The averaged $Q_{2}$ in outer retina $\left(\mathrm{Q}_{a v} \mathrm{O}_{2}\right)$ under light adaption has been characterized by fitting this model to microelectrode data ${ }^{34}$.

From the parameters of the model, the fraction of the outer retina supplied by the RC $\left(F_{r}\right)$ can be derived as ${ }^{35}$

$$
F_{r}=\left[\frac{\left(L_{1}+L_{2}\right)}{2 L}+\frac{\left(P_{L}-P_{C}\right)}{\left(Q_{a v} O_{2} /(D k) L^{2}\right)}\right],
$$

where $P_{c}$ and $P_{L}$ are the $\mathrm{PO}_{2}$ s at the choroid and at $L$, respectively. For the implementation of the model in the present work, $L_{1}, L_{2}$, and $L$ were manually measured from circular scan OCT B-Scan images at ten discrete locations (Supplementary Fig. S4). The averaged values were used in the simulation. It has been experimentally validated that the averaged $\mathrm{PO}_{2}$ in inner retina and $P_{L}$ are well maintained during hypoxia challenge, whereas $P_{c}$ is linearly proportional to arterial oxygen tension $\left(\mathrm{P}_{\mathrm{a}} \mathrm{O}_{2}\right)$ as ${ }^{35}$

$$
P_{c}=0.64 P_{a} O_{2}+1.66 .
$$

Under normoxic light-adapted conditions, $F_{r}$ is usually less than $10 \%$; that is, most of the outer retina is supplied by CC rather than RC. However, because $P_{c}$ decreases in hypoxia, $F_{r}$ increases, and part of $\mathrm{rMRO}_{2}$ supplies the outer retina, which could be calculated as:

$$
\mathrm{O}_{2(\mathrm{RC} \rightarrow \text { Outer retina })}=Q_{a v} \mathrm{O}_{2} \times F_{r} \times m,
$$


where $m$ is the weight of the outer retina. We measured the total weight of the rat retina to be $12 \mathrm{mg}$ and the outer retinal weight $m$ to be $6 \mathrm{mg}$. Table 1 lists the values of characteristic parameters. Some of the parameters necessary to calculate this were measured by us, and some were taken from the literature. Detailed methods to solve the diffusion equation and the simulation of the $\mathrm{PO}_{2}$ profile are provided in Supplementary Information.

\section{Statistics}

Statistical analysis was performed using STATA (StataCorp LP). Two sample T-test was used to test the significance in Figure 3c. Piecewise linear regression was used in Figure $4 \mathrm{~d}-4 \mathrm{f}$. The location of the discontinuity between fitted line segments were determined by minimizing the total square errors (least square error). A fixed effect statistical model was used for the linear regression in Figure $4 \mathrm{~g}-4 \mathrm{i}$ to evaluate the fitted slopes assuming the slopes were the same in different subjects. The intercepts of the fitted line in Figure $4 \mathrm{~g}-4 \mathrm{i}$ were averaged from all the animals.

\section{RESULTS AND DISCUSSION}

We imaged the 3D structure of the rat retina and quantified $\mathrm{rMRO}_{2}$ using vis-OCT (Figure 1a). A focused broadband laser was scanned across the retina to provide transverse $(x, y)$ discrimination. Reflectance at different depths $(z)$ was reconstructed through interference between the reflected light and the reference light. Different contrasts were utilized to calculate $\mathrm{rMRO}_{2}$. Blood flow is the product of the vessel cross-section area and velocity, where the vessel diameter was measured from the tomographic image and the velocity was measured based on the phase variation from the moving blood cells ${ }^{22,23}$. The distinct optical absorption spectra of oxy- and deoxy-hemoglobin provided the contrast for $\mathrm{sO}_{2}$ measurement ${ }^{37,38}$.

Because of the strong attenuation of light in blood within the visible light spectral range, a shadow was cast underneath the vessels (Supplementary Fig. S4). We used an en face slice in the outer retina as a "screen" to capture this "shadow effect" and to create a " $2 \mathrm{D}$ print" of the microvasculature (Figure $1 \mathrm{~b}-1 \mathrm{~d}$ ). We were able to clearly visualize the large retinal vessels as well as the details of the microvasculature. This method does not require a high-density raster scan protocol as reported previously ${ }^{24,39}$, and yet it provides robust labelfree microangiography.

\section{Testing the accuracy of $\mathrm{sO}_{2}$ and blood flow measurements}

First we evaluated the accuracy of flow and $\mathrm{sO}_{2}$ measurement in vitro using vis-OCT (Supplementary Information). For flow measurement verification and calibration, a turbid aqueous solution (1\% Intralipid ${ }^{\mathrm{TM}}$ ) was pumped through a capillary tube by a syringe pump, and then the vis-OCT flow measurements were calibrated against the pump flow settings. For $\mathrm{sO}_{2}$ measurement verification, bovine whole

Table 1 Characteristics of oxygen distribution and consumption in simulation

\begin{tabular}{ll}
\hline $\begin{array}{l}\text { Characteristics of oxygen distribution } \\
\text { and consumption }\end{array}$ & \multicolumn{1}{c}{ Values } \\
\hline$L_{1}(\mu \mathrm{m})$ & 25 (measured) \\
$L_{2}(\mu \mathrm{m})$ & 46 (measured) \\
$L(\mu \mathrm{m})$ & 85 (measured) \\
$\mathrm{PO}_{2}$ at $L, P_{L}(\mathrm{mmHg})$ & $28.8^{35}$ \\
$Q_{a v}\left(\mathrm{~mL} \cdot \mathrm{min}^{-1} \cdot 100 \mathrm{~g}^{-1}\right)$ & $1.8^{34}$ \\
Outer retinal weight $(\mathrm{mg})$ & 6 (unpublished measurement \\
& by Robert A. Linsenmeier) \\
\hline
\end{tabular}

blood samples with controlled $\mathrm{sO}_{2}$ were pumped through a capillary tube and we compared the vis-OCT $\mathrm{sO}_{2}$ quantification with the results derived from blood analyzer readings. The accuracies were within $4.7 \%$ and $4 \%$ relative errors for flow and $\mathrm{sO}_{2}$ measurements, respectively (Supplementary Figs. S5 and S6).

To accomplish flow measurement in vivo, we adopted a dual-circle scanning protocol around the optic nerve head $(\mathrm{ONH})^{23}$. Because retinal blood vessels run radially from the $\mathrm{ONH}$, each circle scanned cross all of the arteries and veins, which allowed us to capture the total retinal blood flow (Figure $2 \mathrm{a}$ ). We repeatedly performed eight dualcircle scans at an A-line rate of $70 \mathrm{kHz}$. The high-speed scanning allowed us to capture the pulsatile profile of the blood flow (Figure 2b). EKG signals were recorded simultaneously to provide correlation between measured retinal blood flow and cardiac cycles. The pulsatile flow pattern from an artery coincided well with the EKG profile, with a slight delay $(\sim 0.1 \mathrm{~s})$ between the peaks of the flow and the QRS complex. This delay was likely caused by the time needed by the sequence of atrioventricular node discharge, ventricular contraction, and the pressure propagation from heart to head. We took a Fourier transform of the pulsatile profile and the distinct peaks from all the arterial flows were consistent, indicating that the frequency of the cardiac cycle was $4.36 \mathrm{~Hz}$ (261.6 $\mathrm{min}^{-1}$ heart rate). We averaged the pulsatile flow readings over the eight dual-circle scans for each vessel and calculated the total arterial and venous flows. The two total blood flows agreed with each other within a measurement precision ( $\pm 0.38 \mu \mathrm{L} \mathrm{min}^{-1}$ averaged from $n=4$ rats) (Supplementary Fig. S7), verifying the consistency between the inward (venous) and outward (arterial) blood flows. The value of total averaged blood flow was 6-8 $\mu \mathrm{L} \min ^{-1}$ ( $n=4$ rats), which agrees well with reported values using the same anesthesia protocol ${ }^{40}$.

Two experimental tests were used to examine the accuracy of our in vivo $\mathrm{sO}_{2}$ measurement. In the first test, we gradually changed the oxygen content in the ventilated air from $21 \%$ to $10 \%$ in six steps. After each adjustment of $\mathrm{O}_{2}$ content, the animals were allowed to adapt to the changed air and re-stabilize for 2 mins. The systemic peripheral arterial oxygenation $\left(\mathrm{spO}_{2}\right)$ was monitored by a pulse oximeter attached to a rear leg of the rats. At each inhalation condition, we measured arterial $\mathrm{sO}_{2}$ by vis-OCT and compared the averaged values with the pulse oximeter $\mathrm{spO}_{2}$ readings (Figure $2 \mathrm{~d}$ ). The linear correlation $\left(R^{2}=0.839\right)$ established the responsiveness of our retinal $\mathrm{sO}_{2}$ measurements to the systemic $\mathrm{sO}_{2}$ changes. We observed a slightly lower $\mathrm{spO}_{2}$ value than the vis-OCT $\mathrm{sO}_{2}$, which may be due to the mild ischemia caused by the mechanical pressure from the sensor clip designed for human use. In the second test, we changed the inhaled oxygen from $21 \%$ to $100 \%$, then back to $21 \%$, and finally to $10 \%$ (Figure 2e). Arterial $\mathrm{sO}_{2}$ was roughly unchanged from $21 \%$ to $100 \%$, but dropped significantly at $10 \%$ oxygen $(0.95 \pm 0.02$ at $21 \%$ oxygen, $0.95 \pm 0.01$ at $100 \%$ oxygen, $0.96 \pm 0.01$ at recovery $21 \%$, and $0.59 \pm 0.03$ at $10 \%$ oxygen); on the other hand, the venous $\mathrm{sO}_{2}$ changed with every change in oxygen content $(0.75 \pm 0.03$ at $21 \%$ oxygen, $0.86 \pm 0.01$ at $100 \%$ oxygen, $0.74 \pm 0.02$ at recovery $21 \%$, and $0.48 \pm 0.01$ at $10 \%$ oxygen). The arterial $\mathrm{sO}_{2}$ changed much less than venous $\mathrm{sO}_{2}$ during hyperoxia because the arterial blood was already well oxygenated under normal air breathing.

Testing the longitudinal monitoring of blood flow, $\mathrm{sO}_{2}$, and $\mathrm{rMRO}_{2}$ The advantage of using vis-OCT to measure $\mathrm{rMRO}_{2}$ is the label-free and non-invasive nature, which allows us to perform longitudinal monitoring of blood flow, $\mathrm{sO}_{2}$, and $\mathrm{rMRO}_{2}$ from the same object. To demonstrate that, we performed a time-course study, in which five 
measurements were taken from the same subjects over two weeks (Supplementary Fig. S8). The standard deviations of four functional parameters measurements were all within $11 \%$ of the mean values (7.4\% for arterial $\mathrm{sO}_{2}, 6.4 \%$ for venous $\mathrm{sO}_{2}, 9 \%$ for blood flow, and $11 \%$ for $\mathrm{rMRO}_{2}$ ).

\section{Retinal metabolic response to systemic hypoxia}

Having characterized the accuracy of blood flow and $\mathrm{sO}_{2}$ measurements, we studied how systemic oxygen tension affects $\mathrm{rMRO}_{2}$ during hypoxia. Although previous studies have shown hemodynamic (increased retinal blood flow) and anatomical vascular changes (increased vessel diameter) under low oxygen supply ${ }^{41}$, a comprehensive observation of how inner retinal oxygen consumption reacts to limited oxygen supply was never reported using a non-invasive approach. In addition, the oxygen supply to the outer retina relies mostly on the choroidal circulation (CC) and little on the RC under light-adapted conditions ${ }^{34,35}$, but how their roles change in supplying the outer retina during hypoxia is still unknown.

We first investigated the retinal vascular anatomical changes under hypoxia (Figure 3). We observed that the major arteries and veins dilated under hypoxia. The average vessel diameter increased by $35 \%$ in arteries $(59.7 \pm 1.5 \mu \mathrm{m}$ during normoxia; $80.8 \pm 2.0 \mu \mathrm{m}$ during hypoxia), and $16 \%$ in veins $(77.4 \pm 2.0 \mu \mathrm{m}$ during normoxia; $90.2 \pm 2.3 \mu \mathrm{m}$ during hypoxia). Under normoxia, the arteries were curved due to a constrictive vascular tone; under hypoxia, as a comparison, straighter arteries indicated relaxation of vascular smooth muscle. In addition, we also observed dilation in smaller arterioles (Figure 3d-3f), which allows more blood flow into the deep retinal capillary network in the outer plexiform layer (OPL).

In order to progressively track the auto-regulatory response, we used a "step-down" hypoxia challenge protocol, in which the inhaled oxygen content was reduced from $21 \%$ (normoxia) to $9 \%$ (hypoxia) in six steps $(21 \%, 19 \%, 16 \%, 14 \%, 11 \%$, and $9 \%)$ as shown in Figure $4 a$. Multiple measurements were taken at each step and the progressing trends of $\mathrm{sO}_{2}$, blood flow, vessel diameter, and $\mathrm{rMRO}_{2}$ were quantified separately ( $n=10$ rats). The entire experiments took less than 30 mins. Heart rate was monitored by the pulse oximeter during the experiments. During air breathing it was $280 \pm 13.3 \mathrm{~min}^{-1}$ (mean and SEM). On average, it increased by about $5 \%$ at an intermediate $\mathrm{O}_{2}$ content of $14 \%$, and returned toward the control value at lower $\mathrm{O}_{2}$ content of $9 \%$.

As expected, both arterial and venous $\mathrm{sO}_{2}$ decreased with reduced oxygen supply (Figure $4 \mathrm{~b}$ ). The venous $\mathrm{sO}_{2}$ decreased almost linearly, while the arterial $\mathrm{sO}_{2}$ decreased more rapidly when the oxygen content was below $14 \%$. Because $\mathrm{PO}_{2}$ is a direct stimulus to autoregulation, we translated our $\mathrm{sO}_{2}$ readings to $\mathrm{PO}_{2}$ based on the haemoglobin dissociation curve (Figure 4b), defined by Hill's equation ${ }^{42}$ with $n=2.8$ and $\mathrm{P} 50=32.9 \mathrm{mmHg}$ :

$$
\mathrm{PO}_{2}=\left(\frac{s \mathrm{O}_{2}}{1-s \mathrm{O}_{2}}\right)^{\frac{1}{n}} \mathrm{P} 50 .
$$

For arteries and veins, the average $\mathrm{PO}_{2} \mathrm{~s}$ were $103.7 \pm 5.8$ and $46.3 \pm$ $1.5 \mathrm{mmHg}$, respectively, and dropped almost linearly to $36.3 \pm 1.2$ and $26.3 \pm 0.8 \mathrm{mmHg}$ at $9 \%$ inhaled $\mathrm{O}_{2}$ (Figure $4 \mathrm{c}$ ). The arteriovenous $\mathrm{sO}_{2}$ difference exhibited a two-segment pattern that decreased slightly when the arterial $\mathrm{PO}_{2}$ was higher than $\sim 60 \mathrm{mmHg}$ and decreased quickly thereafter (Figure $4 \mathrm{~d}$ ). When we examined the progressive trend of vessel diameter with arterial $\mathrm{PO}_{2}$, it also had a similar twosegment pattern where the dilation became more dramatic during severe hypoxia (Figure 4e). A consequence of vessel dilation was the reduction in vascular resistance, which allowed more blood flow (Figure 4f). The discontinuous point of the two segment pattern was observed at a $\mathrm{P}_{\mathrm{a}} \mathrm{O}_{2}$ of $\sim 60 \mathrm{mmHg}$ when the inhaled oxygen was about $16 \%$ (Figure $4 \mathrm{c}$ ). When oxygen was further reduced, the animals were more severely hypoxic, which may cause more dramatic autoregulation. The increased blood flow compensated for the deficiency in $\mathrm{s}_{\mathrm{a}} \mathrm{O}_{2}$ (arterial $\mathrm{sO}_{2}$ ), and the total oxygen delivery (defined by $1.34 \times$ $F_{\text {artery }} \times \mathrm{s}_{\mathrm{a}} \mathrm{O}_{2}$ ) by arterial vessels was actually increased (slope $=$ -0.0029) (Figure $4 \mathrm{~g}$ ). The oxygen extraction fraction (defined by the ratio of arteriovenous $\mathrm{sO}_{2}$ difference divided by $\mathrm{s}_{\mathrm{a}} \mathrm{O}_{2}$ ) increased, indicating that the retina extracted oxygen more efficiently under hypoxia (Figure $4 \mathrm{~h}$ ). Finally, $\mathrm{rMRO}_{2}$ also increased with the decreased $\mathrm{P}_{\mathrm{a}} \mathrm{O}_{2}$ (Figure $4 \mathrm{i}$ ). The slopes in Figure $4 \mathrm{~g}-4 \mathrm{i}$ were significantly different from zero ( $p=<0.001,0.001$, and $<0.001$, respectively), with the scatter contributed largely by the vertical offset of the data from individual animals, each of which exhibited the same trend in slope (illustrated in the inset in Figure 4i). A summary of the slope fitting statistics for oxygen delivery, oxygen extraction fraction and $\mathrm{rMRO}_{2}$ is listed in Table 2.

\section{Balanced oxygen supply from retinal and CCs}

The increased extraction of oxygen during hypoxia was not previously observed. We believe it is a result of increased oxygen supply to the outer retina from the RC when oxygen supply from CC drops (Figure 5a). Such a balance between the RC and CC is not an active compensation during hypoxia; rather, it results from the different responses to oxygen deficiency by the two circulations. The CC has a small arteriovenous $\mathrm{sO}_{2}$ difference and very little autoregulatory response, while the $\mathrm{RC}$ has a large arteriovenous $\mathrm{sO}_{2}$ difference and is well regulated ${ }^{43}$. To determine whether this balancing mechanism could quantitatively account for the increased oxygen extraction from the RC, we compared our experimental results with a simulation model built upon oxygen electrode measurements in the retina ${ }^{34}$. The model quantifies the oxygen consumption in the outer retina, and theoretically predicts the percentage of oxygen supply from RC and CC. It has been experimentally shown that the oxygen consumption does not change significantly (i.e. averaged $\mathrm{PO}_{2}$ does not change significantly) in the inner retina during hypoxia challenge under light adaptation $^{34,35}$. Thus by calculating the oxygen supplied from RC to outer retina, the model can predict the change of $\mathrm{rMRO}_{2}$.

The $\mathrm{PO}_{2}$ profile across the retina is illustrated in Figure $5 \mathrm{~b}$ and $5 \mathrm{c}$. When the systemic $\mathrm{PO}_{2}$ drops, the choriocapillaris $\mathrm{PO}_{2}$ also drops and increases the oxygen gradient from inner retina to outer retina ${ }^{34-36}$. The amount of oxygen delivered from the inner retina to the outer retina can

Table 2 Summary of the slopes of the linear fitting against arterial $\mathrm{PO}_{2}$

\begin{tabular}{|c|c|c|c|c|}
\hline & Slope & Robust SE & $p$ value & [95\% confidence interval] \\
\hline Oxygen extraction fraction (OEF) & -.0013995 & .000274 & $<0.001$ & [-.0019501 to -.0008489$]$ \\
\hline Oxygen delivery (OD) & -.0028637 & .000828 & 0.001 & {$[-.0045277$ to -.0011997$]$} \\
\hline $\mathrm{rMRO}_{2}$ & -.0020111 & .0003709 & $<0.001$ & {$[-.0027563$ to -.0012658$]$} \\
\hline
\end{tabular}


a

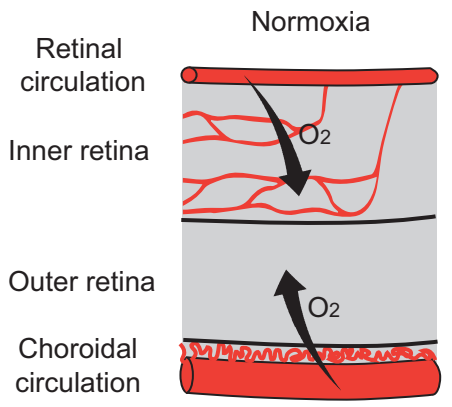

b
C d

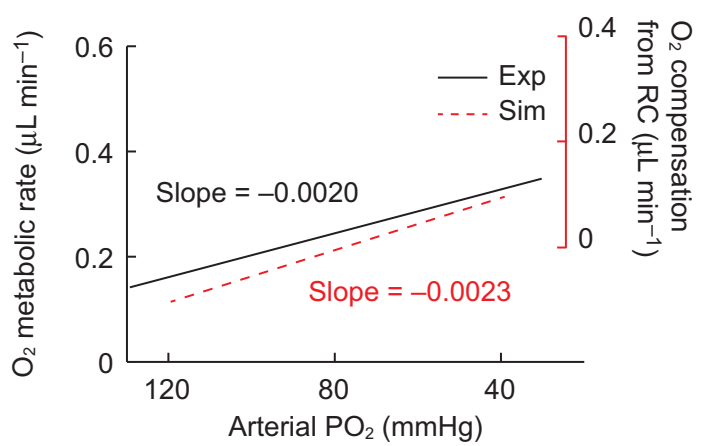

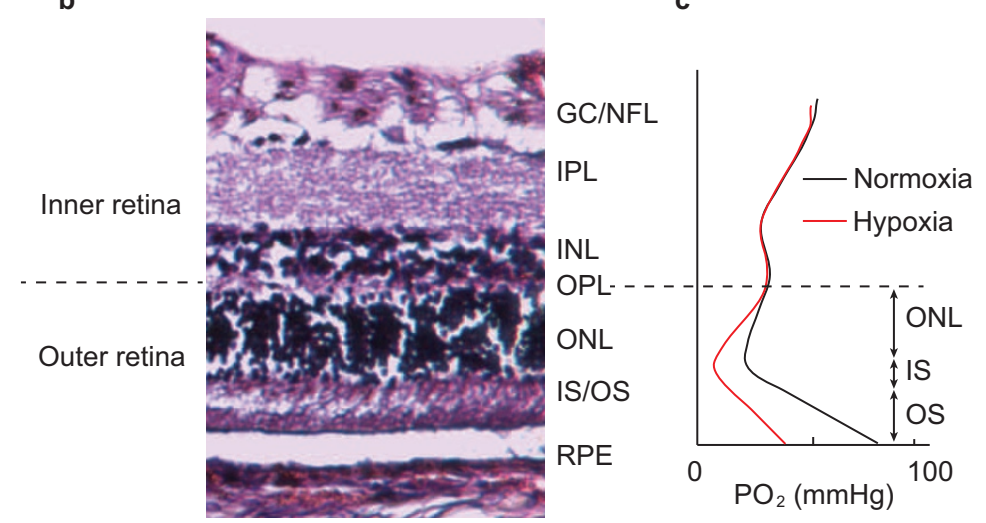

Figure 5 Balance of oxygen supplies between retinal and choroidal circulations under hypoxia. (a) Under systemic hypoxia, the retinal circulation provides more oxygen to the outer retina to compensate the deficit from the choroidal circulation. (b) Anatomical structure of a rat retina. The retinal pigment epithelium (RPE) resides beneath the neural retina, which consists of several defined layers: the outer (OS) and inner segment (IS) of the photoreceptor, and the outer nuclear layer (ONL), outer plexiform layer (OPL), inner nuclear layer (INL), inner plexiform layer (IPL), ganglion cell (GC), and nerve fiber layer (NFL). The boundary between the inner retina and outer retina is the OPL. (c) Simulated $\mathrm{PO}_{2}$ profile across the retina (Supplementary Fig. S9). The inner retinal $\mathrm{PO}_{2}$ is assumed to be constant under the level of hypoxia used here, while the outer retinal $\mathrm{PO}_{2}$ profile changes. The majority of the oxygen is consumed in the IS, where the $\mathrm{PO}_{2}$ reaches a minimum in the outer retina. The oxygen diffuses from both the choroidal and retinal circulations, forming two $\mathrm{PO}_{2}$ gradients toward the IS. This distinct $\mathrm{PO}_{2}$ profile in three segments in the outer retina can be modeled by a one-dimensional oxygen diffusion model. (d) Comparison of the oxygen metabolism from experimental observation and simulation. The experimental oxygen metabolic rate is re-plotted from Figure $4 \mathrm{i}$.

then be calculated by Equations 7-9, in which the averaged outer retina oxygen consumption and $\mathrm{PO}_{2}$ has been previously characterized ${ }^{34}$. Notably, Equation 9 reveals an expected linear relationship between $\mathrm{rMRO}_{2}$ and arterial $\mathrm{PO}_{2}$, based on which we linearly fitted our experimental data. When we compared the simulated change of $\mathrm{rMRO}_{2}$ with the experimental $\mathrm{rMRO}_{2}$ as in Figure $5 \mathrm{~d}$, the trends with decreased arterial $\mathrm{PO}_{2}$ were almost identical (slope $=-0.0023$ in the simulation and -0.0020 in the in vivo experiment). This implies that the apparent increase in the detected oxygen consumption during hypoxia was due to the additional oxygen provided to the outer retina, and that for the range of arterial $\mathrm{PO}_{2}$ in our experiments, inner retinal oxygen consumption actually did not change.

\section{Discussion}

It is well known that RC and CC behave differently under systemic hypoxia ${ }^{5}$. This has been experimentally shown by the direct microelectrode measurements by Linsenmeier and Braun in $\mathrm{cats}^{35}$. They showed that the $\mathrm{PO}_{2}$ in the inner retina was well maintained under hypoxia challenge under light adaption; while the $\mathrm{PO}_{2}$ dropped in the chorioicapillaris. This difference in $\mathrm{PO}_{2}$ results from the fact that blood flow in the RC increases during hypoxia (as we have verified noninvasively), while blood flow in the CC remains unchanged. Although the balance of oxygen supply between RC and CC is somewhat predicted by the diffusion model, noninvasive measurement of consumption of oxygen derived from RC has never been reported. Here we used a step-wise hypoxia challenge to experimentally observe the $\mathrm{rMRO}_{2}$ response to systemic $\mathrm{PO}_{2}$ under light adaption. The noninvasive nature of our approach allows us to continuously monitor the same animal through the entire hypoxia challenge. Counterintuitively, we found that $\mathrm{rMRO}_{2}$ increased with lower systemic $\mathrm{PO}_{2}$, which was explained by the oxygen diffusion model. In contrary, Wanek et al. reported a reduced $\mathrm{rMRO}_{2}$ under hypoxia challenge using fluorescent microsphere to measure blood flow and two photon phosphorescence life time to measure $\mathrm{sO}_{2}{ }^{44}$. Although the reduced arterial $\mathrm{sO}_{2}$ and arteriovenous $\mathrm{sO}_{2}$ difference in our study were consistent with their findings, we observed monotonically increased blood flow under hypoxia; whereas in their results the blood flow increased at moderate hypoxia and returned to the normal level at severe hypoxic challenge. This appears to be the major discrepancy. If we decreased inspired oxygen further, we would expect that eventually the $\mathrm{RC}$ would fail to compensate and see the result observed by Wanek et al.

Translating vis-OCT from rodents to humans requires a few additional considerations. The first one is whether the visible light illumination will induce metabolic change during imaging. It has been shown that transitions between dark and light adaptation cause transient changes in retinal blood flow and oxygen metabolism ${ }^{45-47}$; 
however, constant visible light exposure under light adaptation does not ${ }^{48,49}$. This indicates that vis-OCT is not likely to affect retinal oxygen metabolism when eyes are already light-adapted before imaging. Another practical concern is that the scanning visible light beam may distract eye fixation during imaging. However, this can be overcome by integrating an additional near infrared OCT channel for alignment purpose, where the visible light channel can only be turned on at the time of data acquisition for a short period of time.

The ability to quantify $\mathrm{rMRO}_{2}$ can potentially provide valuable insight into the pathogeneses of various retinal diseases, particularly DR and glaucoma. A key element is to understand the causal relationship between retinal cell degeneration and hemodynamic dysregulation. For example in DR, it is known that endothelial and pericyte disruption occurs in early-stage DR, but how the associated hemodynamic changes are unknown. Some studies showed increased retinal blood flow ${ }^{50,51}$ and suggested that the higher blood flow and high glucose level causes hyperperfusion, which further damages the endothelium and pericytes ${ }^{51}$. On the other hand, contradictory data exist and show that decreased blood flow is one of the earliest changes in diabetic retina ${ }^{52,53}$. The hypothesis is that the loss of pericytes in the early phase of the disease reduces oxygen consumption, which may paradoxically lead to increased oxygenation of the retina. This might create a hyperoxia, resulting in vasoconstriction and reduced blood flow $^{54}$. Similarly, in glaucoma, there exists degeneration of retinal ganglion cells. Although altered blood flow and vasculature were observed in glaucoma, their causal relationship to ganglion cell death remains unknown ${ }^{55}$. Through measuring $\mathrm{rMRO}_{2}$, we will have the opportunity to correlate metabolic function and blood flow, which could potentially reveal the connection between hemodynamic dysregulation and retinal cell degeneration. With improved understanding of retinal metabolic function, improved approaches to early disease detection and therapeutic strategies can be designed.

The ability to quantify oxygen metabolism will expand the applications of OCT to a much broader scope. Vis-OCT provides a convenient approach that allows both blood flow and $\mathrm{sO}_{2}$ quantification with one set of measurements, which otherwise would either be invasive or requires different imaging systems.

\section{CONCLUSIONS}

We demonstrated the capability of vis-OCT to accurately measure $\mathrm{rMRO}_{2}$ and to monitor $\mathrm{rMRO}_{2}$ response subject to systemic oxygen modulation. This method allowed us to monitor retinal function via its oxygen consumption. We experimentally measured the response of $\mathrm{rMRO}_{2}$ to systemic $\mathrm{PO}_{2}$ variations and observed increased oxygen consumption supplied from the RC under hypoxia. This is explained by the balanced oxygen supply between retinal and CC. This method presents a noninvasive way of studying the role of oxygen consumption in various diseases whether the dysregulation of oxygen metabolism is important.

\section{ACKNOWLEDGEMENTS}

We would like to acknowledge the generous financial support from the NIH (Grant Nos 1R01EY019951 and 1R24EY022883) and NSF (Grant Nos CBET1055379 and CBET-1066776). Wenzhong Liu is supported by a HHMI graduate student fellowship. Ji Yi is supported by a Seed Grant from the Illinois Society for Blindness Prevention and a post-doctoral fellowship award from the Juvenile Diabetes Research Foundation (JDRF).

1 Hammes HP. Pericytes and the pathogenesis of diabetic retinopathy. Horm Metab Res 2005; 37: 39-43.
2 Mozaffarieh M, Grieshaber MC, Flammer J. Oxygen and blood flow: players in the pathogenesis of glaucoma. Mol Vis 2008; 14: 224-233.

3 Nowak JZ. Age-related macular degeneration (AMD): pathogenesis and therapy. Pharmacol Rep 2006; 58: 353-363.

4 Caprara C, Grimm C. From oxygen to erythropoietin: relevance of hypoxia for retina development, health and disease. Prog Retin Eye Res 2012; 31: 89-119.

5 Lange CA, Bainbridge JW. Oxygen sensing in retinal health and disease. Ophthalmologica 2012; 227: 115-131.

6 Crosson LA, Kroes RA, Moskal JR, Linsenmeier RA. Gene expression patterns in hypoxic and post-hypoxic adult rat retina with special reference to the NMDA receptor and its interactome. Mol Vis 2009; 15: 296-311.

7 Kaur C, Sivakumar V, Foulds WS. Early response of neurons and glial cells to hypoxia in the retina. Invest Ophthalmol Vis Sci 2006; 47: 1126-1141.

8 Arjamaa O, Nikinmaa M. Oxygen-dependent diseases in the retina: role of hypoxiainducible factors. Exp Eye Res 2006; 83: 473-483.

9 Linsenmeier RA, Braun RD, McRipley MA, Padnick LB, Ahmed J et al. Retinal hypoxia in long-term diabetic cats. Invest Ophthalmol Vis Sci 1998; 39: 1647-1657.

10 Shahidi M, Wanek J, Blair NP, Little DM, Wu T. Retinal tissue oxygen tension imaging in the rat. Invest Ophthalmol Vis Sci 2010; 51: 4766-4770.

11 Berkowitz BA, Kowluru RA, Frank RN, Kern TS, Hohman TC et al. Subnormal retinal oxygenation response precedes diabetic-like retinopathy. Invest Ophthalmol Vis Sci 1999; 40: 2100-2105.

12 Riva CE, Grunwald JE, Petrig BL. Autoregulation of human retinal blood flow. An investigation with laser Doppler velocimetry. Invest Ophthalmol Vis Sci 1986; 27: 1706-1712.

13 Palkovits S, Told R, Schmidl D, Boltz A, Napora KJ et al. Regulation of retinal oxygen metabolism in humans during graded hypoxia. Am J Physiol Heart Circ Physiol 2014; 307: H1412-H1418.

14 Palkovits S, Lasta M, Told R, Schmidl D, Boltz A et al. Retinal oxygen metabolism during normoxia and hyperoxia in healthy subjects. Invest Ophthalmol Vis Sci 2014; 55: 4707-4713.

15 Harris A, Dinn RB, Kagemann L, Rechtman E. A review of methods for human retinal oximetry. Ophthalmic Surg Lasers Imaging 2003; 34: 152.

16 Hardarson SH, Harris A, Karlsson RA, Halldorsson GH, Kagemann L et al. Automatic retinal oximetry. Invest Ophthalmol Vis Sci 2006; 47: 5011-5016.

17 Palsson O, Geirsdottir A, Hardarson SH, Olafsdottir OB, Kristjansdottir JV et al. Retinal oximetry images must be standardized: a methodological analysis. Invest Ophthalmol Vis Sci 2012; 53: 1729-1733.

18 Kristjansdottir JV, Hardarson SH, Halldorsson GH, Karlsson RA, Eliasdottir TS et al. Retinal oximetry with a scanning laser ophthalmoscope. Invest Ophthalmol Vis Sci 2014; 55: 3120-3126.

19 Jiao S, Jiang M, Hu J, Fawzi A, Zhou Q et al. Photoacoustic ophthalmoscopy for in vivo retinal imaging. Opt Express 2010; 18: 3967-3972.

20 Song W, Wei Q, Liu W, Liu T, Yi J et al. A combined method to quantify the retinal metabolic rate of oxygen using photoacoustic ophthalmoscopy and optical coherence tomography. Sci Rep 2014; 4: 6525.

21 Yazdanfar S, Rollins AM, Izatt JA. Imaging and velocimetry of the human retinal circulation with color Doppler optical coherence tomography. Opt Lett 2000; 25: 1448-1450.

22 Zhao Y, Chen Z, Saxer C, Xiang S, de Boer JF et al. Phase-resolved optical coherence tomography and optical Doppler tomography for imaging blood flow in human skin with fast scanning speed and high velocity sensitivity. Opt Lett 2000; 25: 114-116.

23 Wang Y, Bower BA, Izatt JA, Tan O, Huang D. Retinal blood flow measurement by circumpapillary Fourier domain Doppler optical coherence tomography. J Biomed Opt 2008; 13: 064003.

24 Zhi Z, Cepurna W, Johnson E, Shen T, Morrison J et al. Volumetric and quantitative imaging of retinal blood flow in rats with optical microangiography. Biomed Opt Express 2011; 2: 579-591.

25 Yi J, Wei Q, Liu W, Backman V, Zhang HF. Visible-light optical coherence tomography for retinal oximetry. Opt Lett 2013; 38: 1796-1798.

26 Yi J, Li X. Estimation of oxygen saturation from erythrocytes by high-resolution spectroscopic optical coherence tomography. Opt Lett 2010; 35: 2094-2096.

27 Robles FE, Wilson C, Grant G, Wax A. Molecular imaging true-colour spectroscopic optical coherence tomography. Nat Photonics 2011; 5: 744-747.

28 de Villota ED, Carmona MT, Rubio JJ, de Andrés SR. Equality of the in vivo and in vitro oxygen-binding capacity of haemoglobin in patients with severe respiratory disease. Br J Anaesth 1981; 53: 1325-1328.

29 Wang Y, Bower BA, Izatt JA, Tan O, Huang D. In vivo total retinal blood flow measurement by Fourier domain Doppler optical coherence tomography. J Biomed Opt 2007; 12: 041215.

30 Suzuki H, Watkins DN, Jair KW, Schuebel KE, Markowitz SD et al. Epigenetic inactivation of SFRP genes allows constitutive WNT signaling in colorectal cancer. Nat Genet 2004; 36: 417-422.

31 Wang Y, Fawzi AA, Varma R, Sadun AA, Zhang X et al. Pilot study of optical coherence tomography measurement of retinal blood flow in retinal and optic nerve diseases. Invest Ophthalmol Vis Sci 2011; 52(2): 840-845.

32 Boettner EA, Wolter JR. Transmission of the ocular media. Invest Ophthalmol Vis Sci 1962; 1: 776-783.

33 Schwinn DA, McIntyre RW, Reves JG. Isoflurane-induced vasodilation: role of the $\alpha$-adrenergic nervous system. Anesth Analg 1990; 71: 451-459.

34 Lau JC, Linsenmeier RA. Oxygen consumption and distribution in the Long-Evans rat retina. Exp Eye Res 2012; 102: 50-58. 
35 Linsenmeier RA, Braun RD. Oxygen distribution and consumption in the cat retina during normoxia and hypoxemia. J Gen Physiol 1992; 99: 177-197.

36 Ahmed J, Braun RD, Dunn R, Linsenmeier RA. Oxygen distribution in the macaque retina. Invest Ophthalmol Vis Sci 1993; 34: 516-521.

37 Faber DJ, Aalders MC, Mik EG, Hooper BA, van Gemert MJ et al. Oxygen saturationdependent absorption and scattering of blood. Phys Rev Lett 2004; 93: 028102.

38 Bosschaart N, Edelman GJ, Aalders MC, van Leeuwen TG, Faber DJ. A literature review and novel theoretical approach on the optical properties of whole blood. Lasers Med Sci 2014; 29: 453-479.

39 Srinivasan VJ, Radhakrishnan $\mathrm{H}$. Total average blood flow and angiography in the rat retina. J Biomed Opt 2013; 18: 076025.

40 Choi W, Baumann B, Liu JJ, Clermont AC, Feener EP et al. Measurement of pulsatile total blood flow in the human and rat retina with ultrahigh speed spectral/Fourier domain OCT. Biomed Opt Express 2012; 3: 1047-1061.

41 Nagaoka T, Sakamoto T, Mori F, Sato E, Yoshida A. The effect of nitric oxide on retinal blood flow during hypoxia in cats. Invest Ophthalmol Vis Sci 2002; 43: 3037-3044.

42 Hill AV. The combinations of haemoglobin with oxygen and with carbon monoxide. I. Biochem J 1913; 7: 471-480.

43 Pournaras CJ, Rungger-Brändle E, Riva CE, Hardarson SH, Stefansson E. Regulation of retinal blood flow in health and disease. Prog Retin Eye Res 2008; 27: $284-330$

44 Wanek J, Teng PY, Blair NP, Shahidi M. Inner retinal oxygen delivery and metabolism under normoxia and hypoxia in rat. Invest Ophthalmol Vis Sci2013; 54: 5012-5019.

45 Kur J, Newman EA, Chan-Ling T. Cellular and physiological mechanisms underlying blood flow regulation in the retina choroid in health disease. Prog Retin Eye Res 2012; 31: 377-406.

46 Newman EA. Functional hyperemia and mechanisms of neurovascular coupling in the retinal vasculature. J Cereb Blood Flow Metab 2013; 33: 1685-1695.
47 Teng PY, Wanek J, Blair NP, Shahidi M. Response of inner retinal oxygen extraction fraction to light flicker under normoxia and hypoxia in rat. Invest Ophthalmol Vis Sc 2014; 55: 6055-6058.

48 Riva CE, Logean E, Petrig BL, Falsini B. Effect of dark adaptation on retinal blood flow. Klin Monbl Augenheilkd 2000; 216: 309-310.

49 Braun RD, Linsenmeier RA, Goldstick TK. Oxygen consumption in the inner and outer retina of the cat. Invest Ophthalmol Vis Sci 1995; 36: 542-554.

50 Kohner EM, Hamilton AM, Saunders SJ, Sutcliffe BA, Bulpitt CJ. The retinal blood flow in diabetes. Diabetologia 1975; 11: 27-33.

51 Kohner EM, Patel V, Rassam SM. Role of blood flow and impaired autoregulation in the pathogenesis of diabetic retinopathy. Diabetes 1995; 44: 603-607.

52 Feke GT, Buzney SM, Ogasawara H, Fujio N, Goger DG et al. Retinal circulatory abnormalities in type 1 diabetes. Invest Ophthalmol Vis Sci 1994; 35: 2968-2975.

53 Bursell SE, Clermont AC, Kinsley BT, Simonson DC, Aiello LM et al. Retinal blood flow changes in patients with insulin-dependent diabetes mellitus and no diabetic retinopathy. Invest Ophthalmol Vis Sci 1996; 37: 886-897.

54 Wright WS, McElhatten RM, Harris NR. Increase in retinal hypoxia-inducible factor$2 \alpha$, but not hypoxia, early in the progression of diabetes in the rat. Exp Eye Res 2011; 93: 437-441.

55 Flammer J, Orgül S, Costa VP, Orzalesi N, Krieglstein GK et al. The impact of ocular blood flow in glaucoma. Prog Retin Eye Res 2002; 21: 359-393.

cc) (i) (2) This license allows readers to copy, distribute and transmit the Contribution as cc. ${ }_{\mathrm{BY}}$ NC SA long as it is attributed back to the author. Readers are permitted to alter, transform or build upon the Contribution as long as the resulting work is then distributed under this or a similar license. Readers are not permitted to use the Contribution for commercial purposes. Please read the full license for further details at - http:// creativecommons.org/licenses/by-nc-sa/4.0/ 